\title{
Stable Propagation of a Burst Through a One-Dimensional Homogeneous Excitatory Chain Model of Songbird Nucleus HVC
}

\author{
MengRu $\mathrm{Li}^{1}$ and Henry Greenside ${ }^{1}$ \\ ${ }^{1}$ Department of Physics, Duke University, Durham NC 27708-0305
}

(Dated: November 8, 2018)

\begin{abstract}
We demonstrate numerically that a brief burst consisting of two to six spikes can propagate in a stable manner through a one-dimensional homogeneous feedforward chain of non-bursting neurons with excitatory synaptic connections. Our results are obtained for two kinds of neuronal models, leaky integrate-and-fire (LIF) neurons and Hodgkin-Huxley (HH) neurons with five conductances. Over a range of parameters such as the maximum synaptic conductance, both kinds of chains are found to have multiple attractors of propagating bursts, with each attractor being distinguished by the number of spikes and total duration of the propagating burst. These results make plausible the hypothesis that sparse precisely-timed sequential bursts observed in projection neurons of nucleus HVC of a singing zebra finch are intrinsic and causally related.
\end{abstract}

PACS numbers:

\section{INTRODUCTION}

A question of great interest to neurobiology is how animals learn to generate temporal patterns of muscle activation. An example that has been much studied in recent years because of its relevance to human speech [1] and because of the rich variety of possible experiments is how songbirds learn to sing a song by auditory-guided vocal feedback 2]. A young male bird first memorizes the song of an adult male of the same species. Then over many months, over many iterations (more than 50,000 iterations for a zebra finch [3]), and by just listening to his own vocalizations, a young male learns to vary the activation of its respiratory and syringeal muscles until his song is able to match accurately the original memorized song. This is an impressive feat given that an adult song might last several seconds, that there is auditory structure that lasts less than $10 \mathrm{~ms}$, that many syringeal and respiratory muscles need to be coordinated, and that some species of songbirds are able to learn and sing many different songs. Nearly all details of this process remain poorly understood, in particular how the young male memorizes a complex song, and how auditory feedback is used to adjust the pattern of muscle activation until the songbird is able to reproduce accurately the memorized song.

Studies of songbirds have shown that certain anatomically and physiologically distinct brain regions called nuclei are associated with the recognition, learning, and production of song (see Fig. 1). A recent experiment by Hahnloser et al 4] recorded extracellular action potentials (spikes) from neurons in awake singing male zebra finches and found that the neurons in the nucleus HVC 5 that project to the robust nucleus of the arcopallium (abbreviated as RA [5]) have the remarkable properties of firing sparsely and precisely during singing. (In the following, HVC neurons that project to RA will be abbreviated as $\mathrm{HVC}_{\mathrm{RA}}$ neurons.) Typically, each $\mathrm{HVC}_{\mathrm{RA}}$ neuron fires a brief burst of about 3-4 spikes once per song motif with each burst lasting about $6 \mathrm{~ms}$. (A motif is a

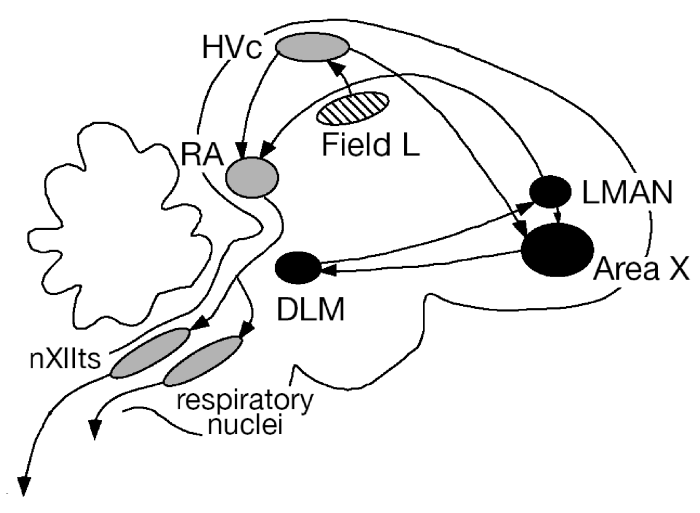

FIG. 1: Schematic anatomical diagram of a sagittal cross section of one hemisphere of a zebra finch brain, showing the major nuclei associated with the learning and production of song. The nucleus HVC plays a central role since it receives auditory and other input, it drives the premotor nucleus RA [4], and it sends information to a parallel anterior forebrain pathway (nuclei X, DLM, and LMAN) that play a role in the learning and maintenance of a song. There are two HVC nuclei, one on the left and one on the right side of the songbird's brain.

cluster of distinct auditory syllables that is repeated as a single pattern and that lasts an average of $0.6 \mathrm{~s}$ for zebra finch songs.) Measurements during successive motifs from a given adult male bird show that each burst from a particular $\mathrm{HVC}_{\mathrm{RA}}$ neuron is aligned with certain acoustic features of the motif to a precision of about $0.7 \mathrm{~ms}$ [4]. These bursts are important to understand since they are believed to provide the temporal framework for organizing the syllables of a song $[6]$.

The observation that each $\mathrm{HVC}_{\mathrm{RA}}$ neuron bursts just once per motif and other data which show that there is a reproducible temporal ordering of $\mathrm{HVC}_{\mathrm{RA}}$ neurons according to when they fire their single burst per motif [4], suggest the hypothesis that bursts propagate along a feedforward network of excitatory $\mathrm{HVC}_{\mathrm{RA}}$ neurons [6]. 
The idea is similar to a path of dominoes such that the falling of one domino causes the next domino in the path to topple. Here, a given $\mathrm{HVC}_{\mathrm{RA}}$ neuron receives one or more bursts from other $\mathrm{HVC}_{\mathrm{RA}}$ neurons that have fired recently during the motif. In response, the given neuron itself fires a burst that helps to activate neurons that fire later during the motif. That each $\mathrm{HVC}_{\mathrm{RA}}$ neuron fires exactly once per motif implies that the connections are feedforward (although this assumption can be weakened if a recurrent burst arrives while some earlier neuron is still in a refractory state [7]). The bursts continue to propagate until the chain ends and the motif stops, or perhaps there is a branch point to a different chain whose dynamics generate a different motif.

While there has been much previous theoretical research concerning when spikes can propagate along excitatory networks of different kinds 8, 9, 10, 11, 12, 13, 14], the songbird experiments on HVC of Hahnloser et al during singing [4] and related experiments by Mooney of bursts in HVC projection neurons during audition [15] motivate asking new theoretical questions about the propagation of a burst through various kinds of networks.

In this paper, we show that a minimal feedforward architecture - a one-dimensional (1d), homogeneous, excitatory chain of non-bursting neurons - can support the stable propagation of a brief high-frequency burst. By studying two kinds of chains, one with leaky integrateand-fire neurons (abbreviated as LIF neurons) and one with more realistic single-compartment conductancebased neurons (abbreviated as HH neurons for HodgkinHuxley like), we show that a burst can propagate in a stable manner that does not require a careful choice of neuronal model nor a careful tuning of model parameters. Depending on the values of parameters such as the maximum synaptic conductance, we find that each kind of $1 \mathrm{~d}$ chain (LIF or HH) has multiple attractors that differ in the number of spikes and in the total width of the propagating burst.

Our calculations are the first to demonstrate that a brief high-frequency burst similar to those observed in HVC can propagate in a stable manner along a simple excitatory chain of neurons. (A similar result was announced independently by Jin and collaborators [7], who studied a more complicated model. We discuss this preprint briefly in Section IV] below.) Our results make plausible one of the simplest explanations of the Hahnloser et al result, namely that the observed bursts of $\mathrm{HVC}_{\mathrm{RA}}$ neurons during singing are intrinsic to $\mathrm{HVC}$ in that external input from other brain regions is not needed to generate the bursts (except the first burst), and in that the bursts are causally related such that one burst initiates the generation of the next burst. Our results also make some predictions such as the existence of multistability (different kinds of propagating bursts can occur in the same chain depending on how the chain is activated), and that transitions from one kind of propagating burst to another kind (differing in the number of spikes and burst width) can occur as parameters are varied. New experimental studies of HVC, especially using optical methods with high time resolution [16] that can examine the spatiotemporal activity of many neurons simultaneously, may help to confirm these ideas.

The rest of this paper is organized as follows. Section! discusses some details of the two classes of mathematical neurons that we use in the $1 \mathrm{~d}$ chains, and how the excitatory synaptic currents are modeled. Section [II presents numerical results mainly for a $1 \mathrm{~d}$ chain of $\mathrm{HH}$ neurons (as opposed to LIF neurons), especially the existence of different attractors corresponding to different kinds of propagating bursts. The paper concludes in Section IV where key results are summarized. We also discuss there how our results are related to alternative hypotheses such as that the bursting arises from propagation through synfire chains [8] or by a central pattern generator (abbreviated as $\mathrm{CPG})$.

In a second paper [17, 18], we will discuss how noise and network heterogeneities affect the propagation of bursts through a synfire chain. This second paper shows that our key conclusions still hold: a brief high-frequency burst can still propagate in a stable manner for a range of noise strengths and for various amounts of heterogeneity. However, we also find that single spikes, even if synchronized across a synfire pool, do not propagate in a stable way for most of the parameters studied, which suggests that short bursts of several spikes are important for achieving robust propagation through realistic synfire chains.

\section{METHODS}

In this section, we discuss some experimental data that justifies the study of a one-dimensional chain of excitatory neurons, and we also point out some of the experimental details that are ignored in our model. We also discuss some details of the integrate-and-fire and conductance-based neuronal models with excitatory synapses that we use in the $1 \mathrm{~d}$ chains. Appendix $\mathrm{A}$ provides further details of equations and parameter values.

\section{A. A Homogeneous 1d Chain of Excitatory Neurons}

The physiological properties of HVC neurons and how they are interconnected within HVC are poorly understood [15, 19, 20, 21, 22] so that it is not possible at this time to develop a quantitative model of the HVC microcircuitry, say at the level of hippocampus models 23]. Researchers have shown [15, 21] that there are at least three main classes of neurons: excitatory neurons that project to nucleus RA, excitatory neurons that project to area $\mathrm{X}$ in the anterior forebrain pathway, and inhibitory interneurons [15, 21] that connect only to other neurons within HVC. Recent paired-electrode recordings by Mooney and Prather [20] have shown that each type 
of HVC neuron makes local connections with the other two types of HVC neurons but details of the connections such as the number, kinds, and strengths of synapses are incompletely known.

Of special importance for this paper is the experimental observation that $\mathrm{HVC}_{\mathrm{RA}}$ neurons synapse with other $\mathrm{HVC}_{\mathrm{RA}}$ neurons 20]. Thus it is possible for a feedforward network of excitatory neurons to exist in HVC, although we emphasize that there is no evidence presently for such a network. Further, there are of order 40,000 $\mathrm{HVC}_{\mathrm{RA}}$ neurons in HVC []], which should be sufficiently many to create a chain whose dynamics spans a motif or even several motifs. (Since the observed bursts in $\mathrm{HVC}_{\mathrm{RA}}$ neurons last $6 \mathrm{~ms}$ [4], a chain of about one hundred pools of neurons would suffice to span a motif of $0.6 \mathrm{~s}$, provided that the bursts do not overlap in time.)

To determine in principle whether the propagation of a burst can explain the experimental data [4, 15], we study one of the simplest possible feedforward networks, namely a one-dimensional homogeneous feedforward chain of identical neurons such that each neuron connects via a single identical excitatory synapse with the next neuron of the chain. The assumption of homogeneity is not realistic biologically but reduces the parameter space of the calculations and increases our ability to understand how the existence and properties of a propagating burst depend on parameters.

Our 1d model leaves out many experimental details (we discuss this further in Section III below), of which two are especially important, the need for multiple pathways and the presence of inhibitory neurons. First, as pointed out by Abeles [8] and others, transmission of information along a one-dimensional chain is not robust since damage to any neuron in the chain can alter or stop the transmission of information. (That HVC is robust is demonstrated by the fact that the adult song of a zebra finch changes little over the life of the bird, even though there is ongoing neurogenesis and hence a steady turnover of $\mathrm{HVC}_{\mathrm{RA}}$ neurons [24, 25].) If some kind of propagation occurs in HVC, there must be parallel pathways.

A widely studied example of a feedforward neuronal architecture with multiple pathways is a synfire chain 8 , 9, 26], see Fig. 2(a). Although there are variations of this architecture, a synfire chain is usually described as a strictly feedforward chain of pools of neurons such that the neurons in one pool project only to neurons of the next pool. Reliable transmission occurs even in the presence of noise or of network heterogeneities because of the multiple paths, and also because each neuron receives spikes from many neurons of the previous pool and the spikes spontaneously become highly synchronized as propagation continues [8, 9]. The arrival of synchronized spikes at the many synapses of a given synfire neuron causes a large postsynaptic current that triggers new spikes with a probability that can approach certainty.

The idea that the sparse brief bursts observed in $\mathrm{HVC}_{\mathrm{RA}}$ neurons are causally connected by sequential firing through some kind of chain has been proposed by
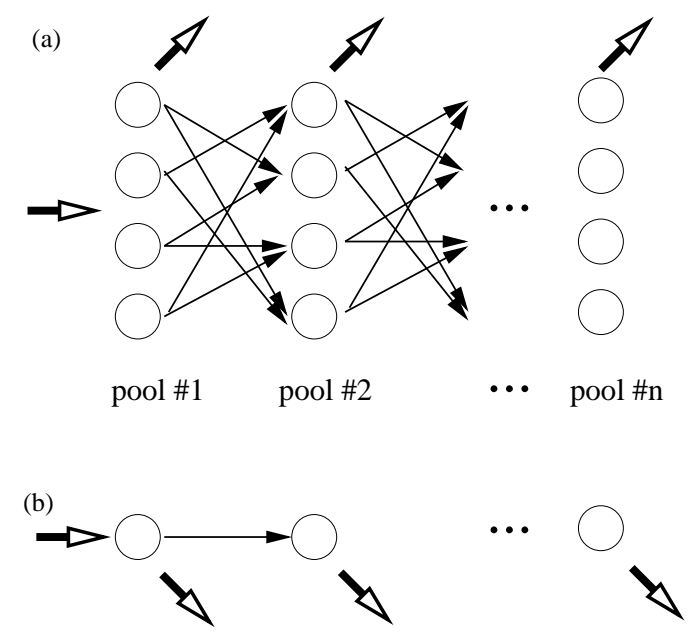

FIG. 2: (a) Schematic diagram of a feedforward synfire chain consisting of successive pools of neurons (vertical column of circles). Each pool contains excitatory $\mathrm{HVC}_{\mathrm{RA}}$ neurons that fire approximately synchronously to activate neurons in the next pool. The horizontal hollow arrow on the left represents input that can initiate the synfire chain. The hollow arrows at the top of each pool indicate efferents that could convey information from a given pool to other brain areas. (b) Schematic diagram of the one-dimensional homogeneous feedforward excitatory chain that we study in this paper. In the biologically unrealistic but theoretically convenient case that all neurons in the synfire chain of (a) are identical, that all neurons in one pool connect to neurons in the next pool in the same way with identical synapses, that axonal delays between pools can be ignored, and there is no noise, the 1d-chain will have dynamics identical to a synfire chain whose spikes have become fully synchronized.

experimentalists 6, 27] and assumed in several theoretical studies [28, 29, 30]. In a recent experiment [26], calcium imaging and timing studies of intracellular recordings have suggested the existence of synfire chains in the visual cortices of mice and cats but no similar imaging study has yet been carried out for songbirds.

The dynamics of our one-dimensional homogeneous chain is equivalent to the dynamics of a homogeneous noiseless synfire chain (homogeneity here means equal numbers of identical neurons per pool all connected in identical ways from one pool to the next) such that all spikes within a given pool have become perfectly synchronized. This is an important observation since the synapse that connects one neuron to the next in the $1 \mathrm{~d}$ chain of Fig. 2(b) then has to be strong to correspond to the many synapses of a neuron in the full synfire chain. In general, noise, heterogeneities, delay times, and imperfect synchronization of spikes cause the dynamics of a synfire chain to differ from that of our 1d chain so that simulations of real synfire chains are needed to understand these effects on the propagation of a burst [7, 17, 18].

A second important experimental detail that we leave out of our model is the presence of the inhibitory HVC interneurons. Our justification for this assumption is sim- 
ply that the function of these interneurons is not known. Proposed mathematical models for sequence generation and sequence recognition incorporate inhibitory neurons in various ways [29, 31, 32, 33] but there is no support yet for these models in the context of the songbird system. We argue that ignoring the inhibitory neurons is a useful first step toward understanding the HVC microcircuitry since the calculations discussed below show that a minimal idealized 1d chain of identical excitatory neurons already suffices to produce a stable propagating burst, in which case other effects such as noise, inhomogeneities, and interneurons could be included perturbatively. Further, some experiments suggest that ignoring the interneurons may not be too severe an assumption. HVC interneurons tend to fire rapidly, chronically, and in approximate synchrony throughout a motif [6], and rapidly and chronically during audition of a bird's own song [15], so that the interneuron spikes might not be closely correlated with the brief sparse bursts of the $\mathrm{HVC}_{\mathrm{RA}}$ neurons. In this case, the main purpose of the interneurons might to prevent runaway excitation of the projection neurons.

We conclude this section with the technical observation that our assumption that the $1 \mathrm{~d}$ chain is homogeneous and that there is no delay along axons (the axons along the chain have effectively zero length) allow the $1 \mathrm{~d}$ chain to be simulated by integrating numerically a single neuron with a single afferent synapse. During the integration, the output of the neuron can be stored in memory and then used as input to the same neuron which then represents the next neuron in a chain. A related idea was used by Reyes 11], who used a single real hippocampal neuron in a slice together with the dynamic clamp technique to simulate a synfire chain consisting of many pools of identical neurons.

\section{B. A Leaky Integrate-and-Fire (LIF) Model of an HVC $_{\text {RA }}$ Neuron}

We studied propagation of bursts along 1d chains consisting of one of two types of neurons, leaky integrateand-fire (LIF) neurons that are described in this section, and more realistic conductance-based Hodgkin-Huxley $(\mathrm{HH})$ neurons that are described in the next section. Neither neuronal model bursts intrinsically when subject to a direct current (DC) external stimulus. Provided that an initial burst is applied to the first neuron of the 1d chain, we find that successive neurons are capable of generating an identical output burst when stimulated itself by a burst.

With respect to conductance-based models, LIF models [34] are attractive since they have fewer parameters, lead to efficient numerical simulations, and are sometimes amenable to analytical methods. The $k$ th LIF neuron of the $1 \mathrm{~d}$ chain $(k \geq 1)$ was modeled as a first-order non- autonomous ordinary differential equation

$$
\tau_{m} \frac{d v^{k}}{d t}=v_{0}-v^{k}+R\left(I_{e}^{k}(t)+I_{S}^{k}(t)\right),
$$

where the parameter $\tau_{m}$ is the capacitive $R C$-time scale of the neuronal membrane, the variable $v^{k}(t)$ is the potential difference across the membrane of the $k$ th neuron in the chain, the external current $I_{e}^{k}(t)$ of the $k$ th neuron is a specified function (in this paper applied only to the first neuron to initiate activity in the chain), the synaptic current $I_{S}^{k}(t)$ arises from spikes in the previous $(k-1)$ st neuron of the chain (see Eqs. (2) and (3) below), the parameter $v_{0}$ is the resting potential toward which the potential $v$ asymptotes in the absence of external and synaptic currents $\left(I_{e}=I_{S}=0\right)$, and the parameter $R$ is the total resistance of the membrane.

Eq. (11) was supplemented with the usual spiking rule that, whenever the potential $v^{k}(t)$ is increasing and crosses a specified threshold value $v_{\text {thresh }}>v_{0}$, the neuron is assumed to have spiked and the potential $v^{k}$ is instantly and discontinuously decreased to a reset value $v_{\text {reset }}<v_{0}$. An absolute refractory period was included in this LIF model by freezing the potential $v^{k}$ to the value $v_{\text {reset }}$ for a time interval $t_{\text {refract }}$ after a spike. We found that including a refractory interval of $t_{\text {refract }}=$ $1 \mathrm{~ms}$ produced only minor qualitative changes to the results obtained in the absence of a refractory period, for example a $1 \mathrm{~ms}$ refractory period expanded by a modest amount the basins of attraction for the different stable propagating bursts discussed in Section III)(see Fig. 6]below). Although $\mathrm{HVC}_{\mathrm{RA}}$ neurons show some accommodation [15], we did not include this detail in our LIF model.

The total postsynaptic current $I_{S}^{k}(t)$ of the $k$ th neuron in the chain was assumed to be a linear accumulation

$$
I_{S}^{k}(t)=\sum_{i} I_{s}\left(t-t_{i}^{k-1}\right)
$$

of postsynaptic currents $I_{s}\left(t-t_{i}^{k-1}\right)$ associated with spikes that occurred in the previous $(k-1)$ st neuron of the chain at times $t_{i}^{k-1}<t$. (Eq. (2) also assumes that there are no time delays arising from the time for a spike to propagate from one neuron to the next.) The time dependence of the synaptic conductivity associated with a single spike at time $t_{i}^{k}=0$ was modeled as a double exponential 34], leading to the following expression for the postsynaptic current per spike:

$$
I_{s}(t, v)=n I_{0}\left(e^{-t / \tau_{1}}-e^{-t / \tau_{2}}\right) .
$$

The slow and fast time constants $\tau_{1}$ and $\tau_{2}$ had respectively the values $1.1 \mathrm{~ms}$ and $0.2 \mathrm{~ms}$ to match roughly the $4.0 \mathrm{~ms}$ onset-to-peak time constants observed in paired intracellular recordings of $\mathrm{HVC}_{\mathrm{RA}}$ neurons [20]. The value $I_{0}=0.3 \mathrm{nA}$ was chosen to give a $1.0 \mathrm{mV}$ peak excitatory post-synaptic potential (EPSP) which is consistent with experiment [20]. The number $n$, which varied from 1 to 32 , indicated the synaptic strength in terms of 
the number of synchronous spikes that a neuron would receive if placed in a uniform synfire chain with $n$ neurons per pool and all neurons of one pool connecting to all neurons in the next pool (with synapses of the same form Eq. (3) ). The range 1 to 32 was found empirically to span the range of stable dynamics, see Fig. [6] below.

An homogeneous chain was then obtained by using for each LIF neuron the same ten parameters $\tau_{m}, v_{0}, R$, $v_{\text {thresh }}, v_{\text {reset }}, t_{\text {refract }}, n, I_{0}, \tau_{1}$, and $\tau_{2}$, and the same function Eq. (3) for the postsynaptic current per spike. A time constant $\tau_{m}=15 \mathrm{~ms}$ was used to approximate the in vivo response time [15], and a membrane resistance of $R=60 \mathrm{M} \Omega$ was chosen to match the impedance of $\mathrm{HVC}_{\mathrm{RA}}$ neurons [15]. Other parameter values used were $v_{0}=-70 \mathrm{mV}, v_{\text {thresh }}=-55 \mathrm{mV}, v_{\text {reset }}=-75 \mathrm{mV}$, and $t_{\text {refract }}=1.0 \mathrm{~ms}$.

The chain of LIF neurons represented by Eqs. (11)-(3) was integrated by using a forward-Euler method [35] with a constant time step of $\Delta t=0.01 \mathrm{~ms}$. (Smaller time steps by factors of four did not lead to significant changes in the results.) The supplementary reset and refractory rules were applied at the end of each time step. For most runs, a zero external current $I_{e}^{k}=0$ was assumed for each neuron of the chain except for the first neuron, for which a step function was used to stimulate a burst. The code was programmed and run using the computational mathematics program Matlab version 6.5 [36].

\section{A Single-Compartment Hodgkin-Huxley Model of an $\mathrm{HVC}_{\mathrm{RA}}$ Neuron}

The second neuronal model that we used to study the propagation of a burst in a $1 \mathrm{~d}$ chain of excitatory neurons was a single-compartment model based on the HodgkinHuxley equations with five representative conductances. The evolution equation for the membrane potential $v^{k}(t)$ of the $k$ th neuron in the chain was

$$
C_{m} \frac{d v^{k}}{d t}=\sum_{i=1}^{5} g_{i}\left(t, v^{k}\right)\left(v_{i}-v^{k}\right)+I_{e}^{k}(t)+I_{S}^{k}(t) .
$$

The symbols have the following meanings: $C_{m}$ is the total membrane capacitance, $g_{i}(t, v)$ is the voltage-dependent conductance of the $i$ th kind of membrane channel, $v_{i}$ is the resting potential for the $i$ th channel, and the currents $I_{e}^{k}(t)$ and $I_{S}^{k}(t)$ have the same meaning as in Eq. (11). Appendix $\mathrm{A}$ gives the details of parameter values and other evolution equations related to the conductances $g_{i}$.

Although more realistic in terms of its time dependence, the HH model Eq. (44) is not necessarily more scientifically appropriate than LIF models since the properties of the various membrane conductances are only partially known for HVC neurons [15, 19, 21, 37], the spatial distribution of the channels in HVC neurons has not been determined (which would be needed to construct accurate multi-compartment models), and little is known about the type, strengths, and locations of synapses in HVC.
We did find that a single-compartment $\mathrm{HH}$ neuron with five conductances is only able to match some features of the experimental HVC data, and it is not clear which experimental details are more important than others to include in the process of fitting a mathematical model to data. For these reasons, and because this paper is concerned whether in principle an HVC-like burst can propagate stably through an excitatory chain, the conductances of the $\mathrm{HH}$ model Eq. (4) were only loosely based on existing HVC data.

Our starting point for choosing the conductances in Eq. (4) was a recent paper by Prinz et al 38], which showed that an eight-dimensional phase space obtained by varying the maximum conductances of eight channels (whose functional properties were obtained from lobster stomatogastric neuronal data) contained a great diversity of dynamical behavior. We chose the functional forms of the leakage, sodium, and potassium channels from this paper and added two other channels as suggested by current-clamp data of HVC neurons [37]: a fourth channel was a low-threshold transient calcium current, and a fifth channel was a calcium-activated potassium current (denoted in the following by the symbol $\mathrm{KCa}$ ). The calcium channel was chosen to activate around $-50 \mathrm{mV}$ and inactivate around $-80 \mathrm{mV}$ with time scales such that the channel could be activated transiently [37, 39]. The KCa conductance activates the potassium current after intracellular calcium concentration rises, with a functional form adopted from Yamada's model [40]. The maximum conductances of all five conductances were adjusted iteratively by hand to produce a short spike width and to generate a spike adaptation with a high initial firing rate as observed by Kubota 37. A reasonably good fit to the Mooney in vivo data (see Fig. 1 of Ref. 15]) unfortunately could not also match Kubota's in vitro spike profiles of Ref [19] and vice versa. (We hope to obtain more complete fits to HVC data by adding more channels and more compartments when more anatomical and physiological data become available.)

The total synaptic current was also assumed to satisfy the linear relation Eq. (2) but an alpha function 34] was used instead of Eq. (3) to approximate the timedependent probability of a channel opening. The excitatory postsynaptic current (EPSC) arising from a spike that occurs at time $t=0$ had the form

$$
I_{s}(t ; v)=g_{s} C\left(\frac{t}{\tau_{s}}\right) e^{-t / \tau_{s}}\left(v_{s}-v\right),
$$

where the constant $C$ with value $e$ normalizes the maximum value of the expression $C\left(t / \tau_{s}\right) \exp \left(-t / \tau_{s}\right)$ to 1 . This normalization makes the parameter $g_{s}$ the maximum conductance, which we varied over the range 0 to $0.55 \mathrm{nS}$. In Eq. (5), the synaptic time constant $\tau_{s}$ was fixed with value $7 \mathrm{~ms}$ and the synaptic reversal voltage $v_{s}$ was set to $120 \mathrm{mV}$ to make the synapse excitatory. Eqs. (4), (2) and (5) together with the evolution equations for gate variables given in Appendix $\mathrm{A}$ were integrated with the Neuron simulation code version 5.6 [41], 
with a constant time step of $\Delta t=0.1 \mathrm{~ms}$.

\section{RESULTS AND DISCUSSION}

\section{A. Results for a 1d Homogeneous Chain of HH Neurons}

The calculations discussed below of one-dimensional homogeneous chains using the LIF or HH neurons of the previous section with excitatory synapses show that a brief high-frequency burst similar to that observed in $\mathrm{HVC}_{\mathrm{RA}}$ neurons during singing 4] can propagate stably over a range of parameter values. These results make more plausible the hypothesis that an excitatory chain of $\mathrm{HVC}_{\mathrm{RA}}$ neurons generates the sparse preciselyaligned bursts observed experimentally [4]. Our results show further that different asymptotic attractors can exist for given parameter values so that hysteresis can occur (different initial conditions can lead to different nontransient dynamics). The fact that the bursts exist as an attractor means that there is a transient time during which properties of the burst such as the number of spikes and burst width (alternatively, the average burst frequency) evolve until the final stable values are obtained. This transient time varies with the initial state used to start the chain and with the choice of parameters.

Since these qualitative conclusions turned out to be similar for LIF and HH neuronal models, we report here results mainly for the $\mathrm{HH}$ models of Section [IC and mention briefly in the next section how the results differ for a chain of LIF neurons. Since the parameter spaces for LIF and HH neurons are high-dimensional (10 parameters for LIF, about 25 parameters for the $\mathrm{HH}$ neurons, and these do not include the choice of the functional form $I_{s}(t, v)$ for the EPSC per spike), to establish our key results we varied only one parameter systematically, namely the maximum synaptic conductance $g_{s}$ of Eq. (5) for $\mathrm{HH}$ neurons, or equivalently the number of synchronous presynaptic spikes $n$ of Eq. (3) for LIF neurons.

Figure [3 shows how the same initial burst of five spikes propagates through a $1 \mathrm{~d}$ excitatory chain of $\mathrm{HH}$ neurons for several different values of the maximum synaptic conductance $g_{s}$. The initial burst was created by using an external current $I_{e}^{1}(t)$ of Eq. (4) for the first neuron in the chain to inject a square pulse of current that caused five spikes to appear in rapid succession. For a synaptic coupling strength $g_{s}$ smaller than about $0.1 \mathrm{nS}$, Fig. Ba shows that the initial burst rapidly dies out by the third neuron of the chain and all spikes disappear. Over a range of stronger couplings $0.2<g_{s}<0.4 \mathrm{nS}$, the initial bursts evolves into a stable invariant propagating burst that can have one to five spikes (columns (b) and (c) of Fig. 31). For stronger couplings $g_{s}>0.4 \mathrm{nS}$, the initial burst is unstable and the number of spikes grows steadily without limit. However, other initial conditions can lead to stable bursts in this range, an example of hysteresis.

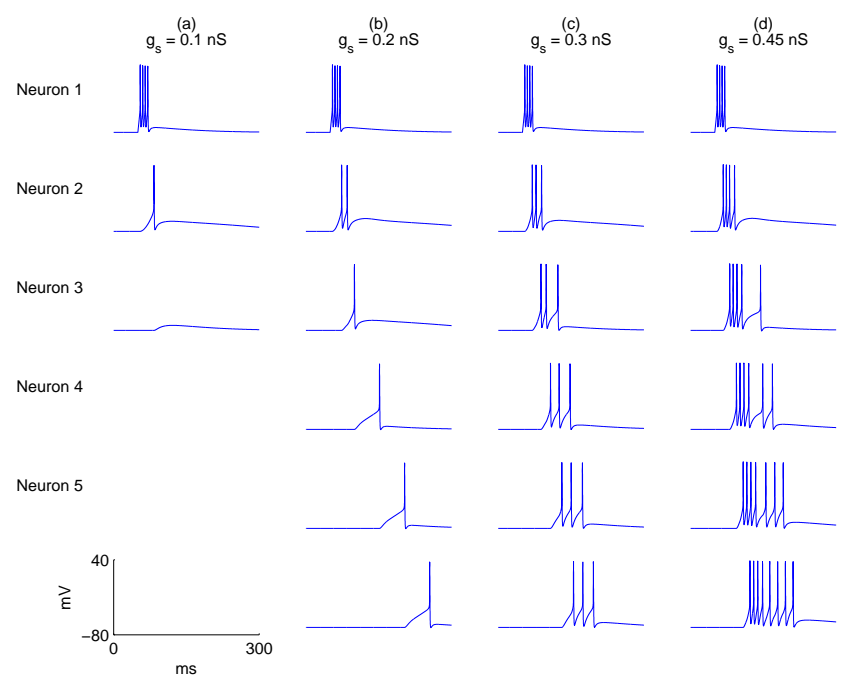

FIG. 3: Propagation of an initial 5-spike burst through an homogeneous one-dimensional excitatory chain of $\mathrm{HH}$ neurons, for different maximum synaptic conductances $g_{s}$ of Eq. (15). Each column is corresponds to a fixed $g_{s}$ value, and successive rows of a given column show the membrane potential $v^{k}(t)$ as a function of time for successive neurons in the chain $(k=1,2, \cdots)$. (a) For a weak synaptic coupling strength $g_{s}=0.1 \mathrm{nS}$, the initial burst decays by the third neuron. (b) For a stronger coupling $g_{s}=0.2 \mathrm{nS}$, the initial bursts decays to an invariant single spike. (c) For $g_{s}=0.3 \mathrm{nS}$, the initial burst evolves to a stable state with three unevenly spaced spikes. (d) For still stronger couplings $g_{s} \geq 0.45 \mathrm{nS}$, the initial burst can be unstable for some initial states and the number of spikes increases steadily.

Fig. 4 provides a more global understanding of the transient dynamics and resulting attractors. In each panel, the vertical axis indicates the number of spikes observed in a burst while the horizontal axis indicates the position $k$ of a neuron along the chain. For a weak synaptic coupling $g_{s}<0.2 \mathrm{nS}$, all initial states decay to zero spikes (not shown in the figure). For a synaptic coupling of $g_{s}=0.3 \mathrm{nS}$ (Fig. 四), initial bursts with three or more spikes decay within four neurons to a common final burst of three spikes. An initial burst with two spikes evolves slightly to an invariant burst with also two spikes, and a similar conclusion holds for an initial burst with a single spike. As the synaptic strength is increased to $g_{s}=0.35 \mathrm{nS}$ (Fig. 40), the number of attractors increases so that stable bursts with one to five spikes are observed depending on the initial state of the first neuron. The transient time is still rather short, with the final number of spikes stabilizing in all cases within three neurons $(k \leq 3)$. Finally for synaptic couplings stronger than about $0.4 \mathrm{nS}$ (Fig. 4. observed depending on the initial state of the first neuron. The unstable bursts all grow at the same rate, with an extra spike appearing for each next neuron traversed.

The transient dynamics of Fig. 4]are presented in somewhat finer detail in Fig. [5 which shows that a non- 
(a)

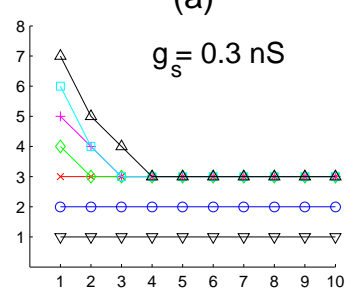

(c)

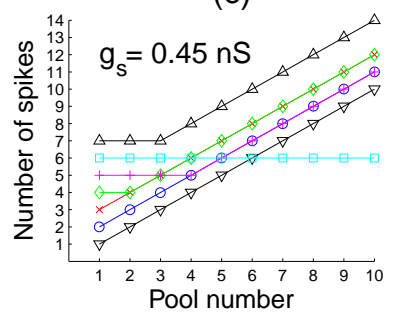

FIG. 4: (Color onlin) The dependence of transient dynamics and resulting attractors of propagating bursts on the initial injecting current magnitude $I_{e}^{1}(t)$ and on the synaptic conductance $g_{s}$. For weak couplings with $g_{s}<0.2 \mathrm{nS}$, all initial states decay to zero (not shown here). (a) For $g_{s}=0.3 \mathrm{nS}$, there are multiple basins of attraction corresponding to asymptotic propagating bursts with 1,2 , or 3 spikes. Transients decay rapidly by the third neuron of the chain. (b) Increasing the synaptic strength to $g_{s}=0.35 \mathrm{nS}$ increases the number of attractors, with final bursts containing 1 to 5 spikes. (c) For a larger synaptic conductance $g_{s}=0.45 \mathrm{nS}$, initial conditions lead to stable or unstable states. An initial burst with 6 spikes will evolve to a slightly different invariant burst with also 6 spikes (squares).

transient propagating bursts is characterized by at least two parameters, their total width and the number of spikes. For example, the same beginning state $B_{1}$ can evolve into three different end points depending on the synaptic coupling strength $g_{s}$ : point $E_{1}$ with three spikes, point $E_{2}$ with 4 spikes and with a slightly longer width of $45 \mathrm{~ms}$, and an unstable state (triangles).

Finally, Fig. 6 indicates the approximate basins of attraction that exist for different initial current amplitudes (horizontal axis) and different maximum synaptic conductances (vertical axis). The plotted numbers indicate the number of spikes observed in the asymptotic state for the specified axis values. This plot shows again how all states decay away for sufficiently weak synaptic coupling, all states are unstable for sufficiently strong coupling, and that stable propagating bursts with 1 to 6 spikes can be found for intermediate coupling strengths. The basins of attraction turn out to be of comparable size except for the tiny basin corresponding to a 6 -spike burst.

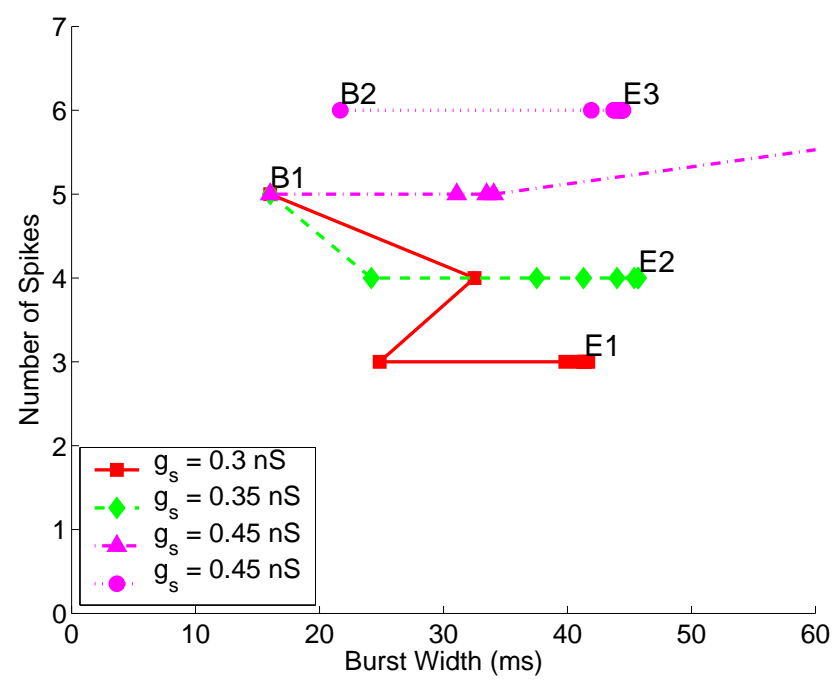

FIG. 5: (Color online) Different synaptic conductances $g_{s}$ lead to different final propagating burst widths and spike numbers, which shows that the total burst duration is another important characteristic of the final attractor. For $g_{s}=0.3 \mathrm{nS}$, the curve of block symbols evolves into the point E1. For $g_{s}=0.35 \mathrm{nS}$, the curve with diamond symbols ends in the point E2. For $g_{s}=0.45 \mathrm{nS}$, the propagation is unstable (upper triangle) except for the initial condition at point B2.

\section{B. Results for a 1d Homogeneous Chain of LIF Neurons}

Our results for homogeneous chains of LIF neurons were qualitatively similar to the above results for chains of $\mathrm{HH}$ neurons and we do not show the corresponding results. Instead of initiating the chain with a current pulse, the first LIF neuron of the chain was initiated with a synaptic current corresponding to a burst of equallyspaced spikes whose times of occurrences $t_{i}^{0}$ were specified as initial data (see Eq. (2)). Similarly, instead of a maximum conductance $g_{s}$ being varied, the synaptic coupling strength $n$ of Eq. (3) was varied between 1 and 32 which we found to span the same kinds of states discussed in Figs. 3]and [6] from a 0 -spike final state to an initial burst that grew without bound in width and in the number of spikes. Because the LIF neurons have no intracellular calcium dynamics, the number of spikes in the final state was primarily determined by the initial spike number. In contrast, the intracellular calcium current of a $\mathrm{HH}$ neuron can boost depolarization and maintain propagating bursts in cohesion so that chains of $\mathrm{HH}$ neurons have bigger basins of attraction for stable bursts.

\section{Other Hypotheses and Related Theory}

We discuss briefly here some other hypotheses and related theoretical work that might explain the data of Hahnloser et al [4] and of Mooney [15] but that do not 


\begin{tabular}{|c|c|c|c|c|c|c|c|}
\hline \multirow{6}{*}{ 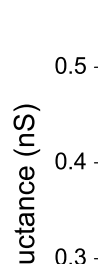 } & $x$ & $\times$ & $x$ & $x$ & $x$ & $x$ & $x$ \\
\hline & $x$ & $x$ & $x$ & $x$ & $x$ & $x$ & $x$ \\
\hline & $x$ & $x$ & $x$ & $x$ & $x$ & 6 & 6 \\
\hline & 2 & 3 & 4 & 5 & 5 & 5 & 5 \\
\hline & 2 & 3 & 4 & 4 & 4 & 5 & 5 \\
\hline & 2 & 3 & 3 & 3 & 3 & 3 & 3 \\
\hline 0 & 2 & 2 & 1 & 2 & 2 & 1 & 1 \\
\hline 0.2 & 1 & 1 & 1 & 1 & 1 & 1 & 1 \\
\hline$\stackrel{\bar{\pi}}{\lessgtr}$ & 0 & 0 & 0 & 0 & 0 & 0 & 0 \\
\hline 0.1 & 0 & 0 & 0 & 0 & 0 & 0 & 0 \\
\hline & 0 & 0 & 0 & 0 & 0 & 0 & 0 \\
\hline & 0.1 & 0.2 & 0.3 & 0.4 & 0.5 & 0.6 & 0.7 \\
\hline
\end{tabular}

FIG. 6: Plot of the number of spikes observed in a final nontransient propagating burst for different initial amplitudes of a square current pulse of fixed duration $20 \mathrm{~ms}$ and for different maximum synaptic conductances $g_{s}$. Different basins of attraction are observed, with 0-spike and unstable bursts having the largest basins, and roughly equal size basins for bursts with 1 to 5 spikes in the final burst.

involve the propagation of bursts through a feedforward network.

While a feedforward network, especially a synfire chain, is one of the simplest concepts that might explain sparse precisely-timed bursting, there is much theoretical work dating back to the 1980 s which shows that a recursive network with inhibitory and excitatory connections is capable of learning and producing different kinds of temporal sequences [32, 42]. These networks generalize the Hopfield attractor model of associative memory [42,43] by allowing non-symmetric couplings between pairs of neurons, and by using two kinds of synapses, "fast" synapses that stabilize a given network state, and "slow" synapses that cause successive transitions between the quasistatic network states. Thus there is no difficulty in principle for a recursive network to store, generate, or learn many temporal sequences of the sort observed in HVC. (We note that other classes of recursive models are possible, e.g., Huerta and Rabinovich [33] discuss a model with neurons that are strictly inhibitory or strictly excitatory, rather than allow any neuron to have inhibitory and excitatory connections with other neurons.)

Asymmetric Hopfield-like recursive networks have several attractive features for modeling HVC dynamics. The HVC inhibitory neurons [15] can be incorporated in a natural way since temporal sequences are stored via neuronal connection strengths $J_{i j}$ that typically have positive (excitatory) and negative (inhibitory) values. Recursive networks that generate sequences can evolve via a simple Hebbian learning rule 32 from densely interconnected neurons (see Figs. 2 and 3 of Ref. 15]) and so are possibly easier to form during maturation of brain tissue than purely feedforward networks. Asymmetric Hopfield models are naturally fault-tolerant and error-correcting so that details of a stored sequence are weakly affected if neurons or synapses are modified, deleted, or added. Finally, a single network of this kind is capable of storing and generating many different temporal sequences, which is consistent with the ability of some songbirds to learn and sing many different songs [2].

However, further work is needed to determine whether existing recursive models 32] are capable of taking into account specific experimental details of $\mathrm{HVC}$ such as the fact that an $\mathrm{HVC}_{\mathrm{RA}}$ neuron fires briefly just once during a one-second-long motif (a neuron is more likely to fire multiple times during a motif if there are recurrent pathways), that time interval between successive bursts is short (estimated to be about $10 \mathrm{~ms}[\underline{6}]$, this is possibly too short for a separation of time scales to exist between slow and fast synapses [32]), that there is a precise alignment of bursts with auditory features during singing [4] and during audition [15], and especially that HVC inhibitory neurons fire tonically while the HVC projection neurons fire sparsely during singing [4] and during audition [15]. (There is no dynamical distinction between inhibitory and excitatory synapses in the recursive models [32].)

Another alternative explanation for the origin and precise alignment of $\mathrm{HVC}_{\mathrm{RA}}$ bursts during singing and during audition is that these bursts are driven by precisely timed external inputs. This possibility is suggested by the fact that other nuclei such as NIF and Uva are known to provide distributed input to $\mathrm{HVC}$, and to all three classes of HVC neurons [44, 45]. However, some experiments suggest that this is a less plausible explanation than intrinsic generation of bursts within HVC. For example, preventing auditory input to HVC by destroying a bird's cochlea (which deafens the bird) or by lesioning nucleus NIF does not prevent a bird from singing, nor does it cause a bird's song to change substantially over time scales of days. Although extracellular recordings of an awake singing bird of the kind reported by Hahnloser et al [4] have not been carried out after deafening or lesioning, the fact that the song does not change immediately suggests that the same pattern of sparse bursts should still be observed in $\mathrm{HVC}_{\mathrm{RA}}$ neurons. Since the inputs to HVC remain incompletely characterized, external driving, or some combination of external driving and intrinsic $\mathrm{HVC}$ circuitry, might be able to explain the observed bursts of $\mathrm{HVC}_{\mathrm{RA}}$ neurons during singing.

A variation of the idea of precise external input driving the bursts in HVC is the possibility that part of HVC acts as a central pattern generator (CPG) that drives the $\mathrm{HVC}_{\mathrm{RA}}$ neurons. For example, the time-ordering of bursts in $\mathrm{HVC}_{\mathrm{RA}}$ neurons observed by Hahnloser et al [4] could arise from a CPG composed of other HVC neurons such that the CPG connects to the $\mathrm{HVC}_{\mathrm{RA}}$ neurons via axons of different lengths and so with different delay times. (The dynamics of the CPG itself could be 
explained as a non-symmetric Hopfield model [32] or a cyclic synfire chain.) A CPG-based model would differ from a synfire chain in that a given burst does not trigger causally the next burst like dominoes falling over along some path. For example, destroying all $\mathrm{HVC}_{\mathrm{RA}}$ neurons that burst at a certain time in a CPG model would not stop the firing of $\mathrm{HVC}_{\mathrm{RA}}$ neurons that normally would burst a short time later. A recent in vitro slice experiment [46] suggests that HVC has intrinsic oscillatory dynamics consistent with the existence of a $\mathrm{CPG}$, but it is not yet known how these slice data relate to the dynamics of $\mathrm{HVC}_{\mathrm{RA}}$ neurons during song.

\section{CONCLUSIONS}

For an idealized one-dimensional homogeneous feedforward chain of excitatory non-bursting neurons, we have shown by numerical calculations that a brief highfrequency burst of two to six spikes can propagate in a stable way for various choices of parameter values. Previous studies of excitatory networks have not addressed the propagation of bursts and so have not been directly relevant for recent experiments concerning the properties of nucleus HVC. Previous studies [9] have also mainly involved networks of LIF neurons (to reduce the computational effort) and so have not taken into account the more complex dynamics and realistic time scales of $\mathrm{HH}$ neurons, especially the effects of a calcium current which experiments have shown to be present in HVC neurons [19, 37]. Our results show that stable bursts exist over a range of parameter values for both LIF and HH neuronal models so that the propagation of stable bursts in a homogeneous chain is not sensitive to the choice of model or of model parameters.

These results make plausible the hypothesis that the sparse precisely-aligned high-frequency brief bursts observed by Hahnloser et al [4] in $\mathrm{HVC}_{\mathrm{RA}}$ neurons during singing are intrinsic (do not need external input) and are causally connected in that one neuron initiates bursting in the next neuron and so on until the end of the chain. However, other explanations of the sparse precise bursts are possible as discussed in Section [IIC and further experiments, especially using methods that can analyze many neurons at once with good time resolution, would be valuable in helping to distinguish feedforward chains from external driving, recursive networks, or other mechanisms.

We have not addressed in this paper why $\mathrm{HVC}_{\mathrm{RA}}$ neurons burst in the first place in, i.e., why information is transmitted as bursts rather than as a population of spikes. (Sparse firing can aid the learning of sequences as pointed out by Fiete et al [47] but this does not require bursts specifically.) There is somewhat of a paradox here in that the commonly posited purpose of a burst is to increase the likelihood of accurate transmission through a noisy network 48, 49], while a similar goal is achieved without bursts by using a synfire chain [8]. Thus if a synfire chain is the correct architecture for $\mathrm{HVC}_{\mathrm{RA}}$ neurons, it is less clear why bursts are needed, especially since HVC has much less noise than cortical neurons and HVC synapses are substantially stronger than cortical synapses [20]. Unpublished calculations by the authors [17, 18] that extend the present paper to noisy heterogeneous synfire chains do suggest that bursts are important for achieving robust propagation even for a synfire architecture.

We finish this paper by summarizing some consequences of the chain hypothesis and of our calculations for recent and future songbird experiments.

1. A simple consequence of the feedforward nature of an excitatory chain is that an experimentalist in principle should be able to initiate singing of an arbitrary contiguous segment of a motif. For example, if it is possible to stimulate an intermediate part of a chain in such a way that a burst begins to propagate toward the end of the chain, a songbird might then sing a motif that starts somewhere in the middle and that then continues to its end. Similarly, physically terminating the chain at some intermediate point (say with a local lesion) could cause a motif to terminate before its usual endpoint, or some combination of stimulating in the middle of the chain and lesioning later in the chain could be implemented in which case the motif could start somewhere in the middle and terminate prematurely.

Electrical stimulation of HVC via a single extracellular electrode in an awake behaving bird does not initiate singing [50, 51, 52] but instead resets a motif to its beginning (if the stimulus occurs while a bird was singing). The failure to initiate the singing of a motif, or part of a motif, does not rule out the existence of an excitatory chain but instead could imply that a carefully-arranged pattern of input spikes might be needed to initiate activity at some point in the chain, especially if the network is a synfire chain for which most neurons in a pool must fire in near synchrony for neurons in the next pool to fire. Identifying the location of $\mathrm{HVC}_{\mathrm{RA}}$ neurons in a chain by, say, optical imaging would be a valuable prior step that could suggest how to work out an electrical or photo-uncaging protocol [53] that could initiate a chain at an arbitrary point along its length, or could suggest how to lesion neurons that would terminate propagation of a burst hence a motif prematurely.

2. Our calculations suggest that a homogeneous chain based on LIF or HH neuronal models should generally have multiple basins of attraction. Different kinds of asymptotic stable bursts differing in the number of spikes and in their total duration should be observed in nucleus HVC for different values of synaptic strength and other neuronal parameters. Further, hysteresis can be expected such that, for 
identical experimental conditions, different initial states will evolve to different kinds of asymptotic bursts.

Hysteresis under fixed experimental conditions can be explored by initiating different kinds of bursts. This may be possible if the neurons at the beginning of the chain can be identified and an appropriate stimulation protocol worked out (see points 1 and 3).

Most nonlinear network models, including asymmetric Hopfield models and CPGs, also have multiple basins of attraction so this is not a distinguishing property of chain models. However, the number and types of basins of attraction, and how these basins vary under the influence of pharmaceuticals that alter synaptic strengths, may lead to predictions that distinguish feedforward from recursive models.

3. Chains of neurons, like the neuronal models that make up the chains, are driven dissipative systems so that initial states will generally evolve through a transient before settling into an asymptotic behavior [54]. Properties of a burst such as its number of spikes and its set of interspike intervals will evolve over time, with the transient time depending on the choice of initial condition (how far the initial state is from the attractor) and on neuronal parameters. Our results such as Fig. 4 for an homogeneous $\mathrm{HH}$ neuronal model suggest that transient times can in fact be rapid, just four or fewer successive neurons. Fig. 2 of Hahnloser et al [4] does not show evidence of transient behavior since later bursts do not seem to be statistically different from earlier bursts during the same motif. However, only a small number (about 20) of $\mathrm{HVC}_{\mathrm{RA}}$ neurons have been sampled to date in singing birds, and neurons that burst at the beginning of a motif have not been found so further study involving more neurons would be worthwhile. It may also be the case that the songbird brain has evolved in such a way as to reduce or eliminate the duration of transients, for example by starting a chain with a state close to the asymptotic burst, or by using inhibitory neurons to accelerate convergence to synchronized firing as bursts propagate from projection neuron to projection neuron [55].

4. A one dimensional chain is not robust since propagation terminates if any neuron in the chain dies. A more realistic synfire chain must have at least two neurons in a pool to be robust, and the neurons in a pool must synchronize their spikes to some extent to achieve reliable transmission from pool to pool [8]. A synfire chain hypothesis thus predicts that there must be more than one $\mathrm{HVC}_{\mathrm{RA}}$ neuron bursting at any give time (these are the neurons that belong to the same pool) and further that neurons that burst at about the same time should have nearly synchronous bursts. Thus Fig 2 of Ref. [4] may be incomplete and a high-resolution optical study of HVC (or possibly a many-electrode study) during the singing of a motif or during the audition of a bird's own song [15] may reveal multiple $\mathrm{HVC}_{\mathrm{RA}}$ neurons firing bursts in synchrony.

Abeles has pointed out that there is an inverse relation between the number of neurons needed in a synfire pool for reliable propagation and the average strength of the synapses between pools [8]. Using paired intracellular recordings, Mooney and Prather 20] have shown that synapses between $\mathrm{HVC}_{\mathrm{RA}}$ neurons are stronger than mammalian cortical neurons by about an order of magnitude (the average EPSP magnitude is about $2 \mathrm{mV}$ compared to about $0.2 \mathrm{mV}$ in cortex) so chains in HVC would be expected to have about an order of magnitude fewer neurons in a pool. Given Abeles's estimate of about 50-100 neurons in a cortical pool, perhaps ten or fewer neurons might be needed in an HVC synfire pool. Assuming that the HVC bursts of average duration $6 \mathrm{~ms}$ are non-overlapping, a zebra finch motif of duration $0.6 \mathrm{~s}$ would require about $0.6 / 0.006 \approx 100$ successive pools to span the motif, so a synfire chain in HVC might involve about $100 \times 10 \approx 1,000$ neurons. This is a much smaller number than the $40,000 \mathrm{HVC}_{\mathrm{RA}}$ neurons estimated to exist in HVC. This suggests that a synfire chain might be too simplistic an architecture to justify the large number of $\mathrm{HVC}_{\mathrm{RA}}$ neurons.

5. It is worth noting that a synfire chain architecture can explain how an overall precise timing can be maintained in HVC even though there is a steady and substantial turnover of neurons in HVC throughout the life of the songbird [24, 25]. Since perfect synchronization of a pool is not needed to guarantee transmission of information through the next pool, a fraction of neurons in a given pool can alter their properties or fail without degrading the transmission of information or its timing.

In conclusion, our calculations support the hypothesis that the sparse bursts observed experimentally in $\mathrm{HVC}_{\mathrm{RA}}$ neurons during singing can be understood as the propagation of bursts through an excitatory feedforward synfire chain. However, further experiments and computational studies are needed to confirm this hypothesis and to rule out the competing hypotheses discussed in Section IIIC Especially interesting in the near term would be to understand some of the quantitative details of the experiments, for example the production of a brief $6 \mathrm{~ms}$ burst of four spikes corresponding to a frequency of about $600 \mathrm{~Hz}$ that is consistent with the known properties of HVC neurons and of the HVC microcircuitry [20]. Although our results show that a brief burst can propagate in a stable manner, we and others [7] have not been able to construct neuronal models, or simple networks of such 
models, that burst as rapidly and as briefly as actual $\mathrm{HVC}_{\mathrm{RA}}$ neurons.

Note: Toward the completion of this paper, the authors received a preprint by Jin et al whose results overlap with the present paper (the preprint is related to a recent conference presentation [7]). These authors also investigated whether brief high-frequency bursts analogous to those observed in $\mathrm{HVC}_{\mathrm{RA}}$ neurons could propagate through a purely excitatory network, and they too found that this was possible for a range of parameters, thereby supporting the hypothesis that the bursting of $\mathrm{HVC}_{\mathrm{RA}}$ neurons could be intrinsic to HVC and causal.

The main difference of the present paper with the preprint is that we explored whether bursts could propagate under the simplest circumstances of a 1d, homogeneous, noiseless, purely-feedforward chain using simple neuronal models. Jin et al studied a more complicated generalized (not purely feedforward), heterogeneous, noisy, purely excitatory synfire chain with many neurons per pool. Jin et al also used a more complicated two-compartment conductance-based neuronal model that included a low-threshold potassium channel that provided a strong spike-frequency adaptation similar to that observed experimentally. (This helped to create brief high-frequency bursts.) They also included calcium dynamics in the dendrite compartment that could lead to the firing of calcium spikes. Jin et al were able to show that adding the low-threshold potassium channel and calcium dynamics made the propagation of bursts through their generalized synfire chain more robust (one did not have to tune parameters so carefully to obtain a stable propagating burst). They also studied how jitter in the burst evolved along the length of the synfire chain, which we have also done in a forthcoming paper with a somewhat different model [17].

Given that our calculations and those of Jin et al have left out the role of inhibitory neurons and were based on a limited knowledge of HVC neuronal properties and of the HVC microcircuitry (e.g., there is no evidence yet for a synfire chain in $\mathrm{HVC}$, nor have the conductances of HVC neurons been fully characterized), both calculations should be considered as modest but complementary and useful steps toward understanding possible mechanisms for the dynamics of HVC during singing.

\section{APPENDIX A: HODGKIN-HUXLEY EQUATIONS AND PARAMETERS}

The single-compartment HH model of Eq. (4) had the following five representative currents $I_{i}(t)$ :

$$
\begin{aligned}
I_{\text {leak }} & =\bar{g}_{L}\left(v-E_{L}\right), \\
I_{\mathrm{Na}} & =\bar{g}_{\mathrm{Na}} m^{3} h\left(v-E_{\mathrm{Na}}\right), \\
I_{\mathrm{K}} & =\bar{g}_{\mathrm{k}} n^{4}\left(v-E_{\mathrm{K}}\right), \\
I_{\mathrm{Ca}} & =\bar{g}_{\mathrm{Ca}} m_{1}^{2} h_{1}\left(v-E_{\mathrm{Ca}}\right), \\
I_{\mathrm{KCa}} & =\bar{g}_{\mathrm{KCa}} n_{1}^{3}\left(v-E_{\mathrm{KCa}}\right),
\end{aligned}
$$

with maximum conductances per unit area y (units of $\mathrm{mS} / \mathrm{mm}^{2}$ ) given

$\bar{g}_{L}=0.003, \quad \bar{g}_{\mathrm{Na}}=1.0, \quad \bar{g}_{\mathrm{K}}=0.22, \quad \bar{g}_{\mathrm{Ca}}=0.031$,

\begin{tabular}{|c|c|c|c|c|c|c|}
\hline & $m$ & $n$ & $h$ & $m_{1}$ & $h_{1}$ & $n_{1}$ \\
\hline$v_{0} \mathrm{mV}$ & -25.5 & -12.3 & -60 & -50 & -80 & \\
\hline$v_{1} \mathrm{mV}$ & 5.29 & 11.8 & -15 & 5 & -5 & \\
\hline$v_{2} \mathrm{mV}$ & -120 & -28.3 & -62.9 & -50 & -80 & -35 \\
\hline$v_{3} \mathrm{mV}$ & 25 & 19.2 & 10 & 10 & 10 & 10 \\
\hline$t_{1} \mathrm{~ms}$ & -1.26 & -6.4 & 1.1 & 20 & 60 & 30 \\
\hline$t_{2} \mathrm{~ms}$ & 1.32 & 7.2 & 0 & 10 & 10 & 10 \\
\hline
\end{tabular}

and corresponding reversal potentials (units of $\mathrm{mV}$ ):

$$
E_{L}=-70, \quad E_{\mathrm{Na}}=50, \quad E_{\mathrm{K}}=-80, \quad E_{\mathrm{Ca}}=120,
$$

TABLE I: Parameter values for the Hodgkin-Huxley model Eq. (4) with currents Eq. (A1) and gating equations Eqs. A5 - A7.

The currents Eqs. A1 depend on the membrane voltage $v(t)$ and on gating variables $m, h, n, m_{1}, h_{1}$, and $n_{1}$ that determine the probability as a function of time for certain channel subunits to be open. All the gating variables $x(v)$ except the KCa activation variable $n_{1}$ obey the evolution equation

$$
\tau(v) \frac{d x}{d t}=x_{\infty}(v)-x
$$

with the asymptotic value $x_{\infty}(v)$ having the sigmoidal form

$$
x_{\infty}(v)=\left(1+e^{-\left(v-v_{0}\right) / v_{1}}\right)^{-1} .
$$

The time constant $\tau(v)$ has the form

$$
\tau(v)=t_{2}+t_{1}\left(1+e^{-\left(\left(v-v_{2}\right) / v_{3}\right)}\right)^{-1}
$$

for the standard $\mathrm{HH}$ channels $m, h$, and $n$, and the form

$$
\tau(v)=t_{2}+t_{1} \exp \left[-\left(\frac{v-v_{2}}{v_{3}}\right)^{2}\right]
$$


for the Ca channels $m_{1}$ and $h_{1}$ and $\mathrm{KCa}$ channel $n_{1}$. Table A gives the parameter values that we used in these various expressions.

The KCa activation variable $n_{1}$ obeys the evolution equation

$$
\tau_{\mathrm{KCa}} \frac{d n_{1}}{d t}=f\left([\mathrm{Ca}]_{i}\right)-n_{1},
$$

where the expression $[\mathrm{Ca}]_{i}$ denotes the average intracellular calcium concentration and the function $f$ is given by

$$
f\left([\mathrm{Ca}]_{i}\right)=\frac{[\mathrm{Ca}]_{i}}{\left([\mathrm{Ca}]_{i}+0.001\right)(1+\exp (-(v+40) / 20))} .
$$

The intracellular calcium concentration accumulates when the low threshold calcium channel is open and calcium ions flow from extracellular solution into intracellular solution. The phenomenological model for $[\mathrm{Ca}]_{i}$ was taken from Ref. [56]

$$
[\mathrm{Ca}]_{i}=\left([\mathrm{Ca}]_{i 0}-[\mathrm{Ca}]_{i}-F *\left(I_{c a}\right)\right) / \tau_{C a},
$$

where the equilibrium Calcium concentration $[\mathrm{Ca}]_{i 0}$ had the value $.00005 \mathrm{mM}$ and the current-density factor $F$ had the value $3 \mathrm{mM}-\mathrm{cm}^{2} / \mathrm{mA}$, and the time constant $\tau_{C a}$ had the value $200 \mathrm{~ms}$. The KCa channel was chosen to imitate a low-threshold transient calcium current observed in HVC neurons by Kubota [19].

\section{ACKNOWLEDGMENTS}

The authors thank Richard Mooney, Jonathan Prather, Melissa Coleman, Michale Fee, Stephen Shea, George Augustine, and Paul Tiesinga for helpful discussions, and Craig Henriquez and Duke University's Center for Neural Engineering for providing support for one of the authors (M. Li).
[1] A. J. Doupe and P. K. Kuhl, Annu. Rev. Neurosci. 22, 567 (1999).

[2] C. K. Catchpole and P. J. B. Slater, Bird Song: Biological themes and variations (Cambridge University Press, New York, 1995).

[3] F. Johnson, K. Soderstrom, and O. Whitney, Behavioural Brain Research 131, 57 (2002).

[4] R. H. R. Hahnloser, A. A. Kozhevnikov, and M. S. Fee, Nature 419, 65 (2002).

[5] A. Reiner, D. J. Perkel, C. V. Mello, and E. D. Jarvis, Ann. N. Y. Acad. Sci. 1016, 77 (2004).

[6] M. S. Fee, A. Kozhevnikov, and R. H. R. Hahnloser, Ann. N. Y. Acad. Sci. 1016, 153 (2004).

[7] D. Jin, F. Ramazanoglu, and H. S. Seung (2005), society for Neuroscience Abstracts No. 753.15.

[8] M. Abeles, Corticonics: Neural Circuits of the Cerebral Cortex (Cambridge University Press, Cambridge, England, 1991).

[9] M. Diesmann, M.-O. Gewaltig, and A. Aertsen, Nature 402, 529 (1999).

[10] R. Oşan, R. Curtu, J. Rubin, and B. Ermentrout, J. Math. Biol. 48, 243 (2004).

[11] A. D. Reyes, Nature Neuroscience 6, 593 (2003).

[12] D. Golomb and Y. Amitai, J. Neurophysiol. 78, 1199 (1997).

[13] V. Litvak, H. Sompolinsky, I. Segev, and M. Abeles, Journal of Neuroscience 23, 3096 (2003).

[14] T. P. Vogels and L. E. Abbott, J. of Neuroscience 25, 10786 (2095).

[15] R. Mooney, J. Neurosci. 20, 5420 (2000).

[16] K. Ohki, S. Chung, Y. H. Ch'ng, P. Kara, and R. C. Reid, Nature 433, 597 (2005).

[17] M. Li and H. Greenside (2006), in preparation.

[18] M. Li, Ph.D. thesis, Duke University (2006).

[19] M. Kubota and I. Taniguchi, J. Neurophysiol. 80, 914
(1998).

[20] R. Mooney and J. F. Prather, Journal of Neuroscience 25, 1952 (2005)

[21] P. Dutar, H. M. Vu, and D. J. Perkel, J. Neurophysiol. 80, 1828 (1998).

[22] J. M. Wild, M. N. Williams, G. J. Howie, and R. Mooney, Journal of Comparative Neurology 483, 76 (2005).

[23] R. D. Traub, J. G. R. Jefferys, and M. A. Whittington, Fast Oscillations in Cortical Circuits (Bradford Books, New York, 1999).

[24] F. Nottebohm, J Neuroscience 22, 624 (2002).

[25] N. Wang, P. Hurley, T. Pytte, and J. R. Kirn, J. Neurosci. 22, 10864 (2002).

[26] Y. Ikegaya, G. Aaron, R. Cossart, D. Aronov, I. Lampl, D. Ferster, and R. Yuste, Science 304, 559 (2004).

[27] A. Leonardo and M. S. Fee, Journal of Neuroscience 25, 652 (2005).

[28] K. Doya and T. J. Sejnowski, in Central Auditory Processing and Neural Modeling, edited by Poon and Brugge (Plenum Press, New York, 1998).

[29] H. D. I. Abarbanel, L. Gibb, G. B. Mindlin, and S. Talathi, J. Neurophysiol. 92, 96 (2004).

[30] L. Berger, Master's thesis, Institute of Neuroinformatics, University/ETH Zürich (2004).

[31] P. J. Drew and L. F. Abbott, J. Neurophysiol. 89, 2697 (2003).

[32] D. Kleinfeld and H. Sompolinsky, in In Methods in Neuronal Modeling: From Synapse to Networks, edited by C. Koch and I. Segev (MIT Press, Cambridge, MA, 1989), pp. 195-246.

[33] R. Huerta and M. Rabinovich, Phys. Rev. Lett. 93, 238104 (4 pages) (2004).

[34] P. Dayan and L. F. Abbott, Theoretical Neuroscience: Computational and Mathematical Modeling of Neural Systems (MIT Press, 2001). 
[35] D. Kincaid and W. Cheney, Numerical Analysis (Brooks/Cole, Pacific Grove, California, 1996), 2nd ed.

[36] MathWorks, Matlab, http://www.mathworks.com/produ

[37] M. Kubota and N. Saito, Journal of Physiology 440, 131 (1991).

[38] A. A. Prinz, C. P. Billimoria, and E. Marder, J. Neurophysiol. 90, 3998 (2003).

[39] J. Huguenard and D. McCormick, J. Neurophysiology 68, 1373 (1992).

[40] C. Koch and I. Segev, eds., Multiple Channels and Calcium Dynamics (MIT, 1998).

[41] M. Hines, J. W. Moore, and T. Carnevale, Neuron, http://neuron.duke.edu/.

[42] J. Hertz, A. Krogh, and R. G. Palmer, Introduction to the Theory of Neural Computation, no. I in Santa Fe Institute Studies in the Sciences of Complexity (Westview, Boulder, CO, 1991).

[43] J. J. Hopfield, Proc. Nat. Acad. Sci. 79, 2554 (1982).

[44] M. J. Coleman and R. Mooney, Journal of Neuroscience 24, 7251 (2004).

[45] M. J. Rosen and R. Mooney, J. Neurophysiol. 95, 1158
(2006).

[46] M. M. Solis and D. J. Perkel, J. Neuroscience 25, 2811 matla(18 $\emptyset 05)$.

[47] I. R. Fiete, R. H. R. Hahnloser, M. S. Fee, and H. S. Sueng, J. Neurophysiol. 92, 2274 (2004).

[48] J. E. Lisman, Trends Neurosci. 20, 38 (1997).

[49] L. M. de la Prida, N. Stollenwerk, and J. V. SanchezAndres, Physica D 110, 323 (1997).

[50] E. T. Vu, M. E. Mazurek, and Y.-C. Kuo, J. Neuroscience 14, 6924 (1994)

[51] E. T. Vu, M. F. Schmidt, and M. E. Mazurek, J. Neuroscience 18, 9088 (1998).

[52] D. S. Vicario and H. B. Simpson, J. Neurophys. 73, 2602 (1995).

[53] A. Losonczy and J. C. Magee, Neuron 50, 291 (2006).

[54] P. Bergé, Y. Pomeau, and C. Vidal, Order Within Chaos (John Wiley \& Sons, New York, 1984).

[55] N. Kopell and B. Ermentrout, PNAS 101, 15482 (2004).

[56] G. Turrigiano, G. Lemasson, and E. Marder, J Neuroscience 15, 3640 (1995). 